\title{
IMAGEM ORBITAL NA IDENTIFICACÃO DE RESPOSTAS TÉRMICAS COM DIFERENTES PADRÕES DE USO E COBERTURA NO MUNICÍPIO DE MOJUÍ DOS CAMPOS, PARÁ
}

Thiago da Silva Soares ${ }^{1}$, Carlos Benedito Barreiros Gutierrez ${ }^{2}$, Dione Margarete Gomes Gutierrez ${ }^{3}$, Altem Nascimento Pontes ${ }^{4}$, Leonardo Sousa dos Santos ${ }^{5}$

${ }^{1}$ Engenheiro Ambiental, Universidade do Estado do Para (UEPA). Discente de Especialização do Curso de Geoprocessamento Aplicado, Faculdade Ideal (FACl), Belém, Pará, Brasil. e-mail: thiagoseraos@gmail.com.

${ }^{2}$ Mestre em Ciências Ambientais. Universidade do Estado do Pará (UEPA), Belém, Pará, Brasil.

${ }^{3}$ Mestranda em Ciências Ambientais, Universidade do Estado do Pará (UEPA), Brasil.

${ }^{4}$ Professor e Pesquisador do Programa de Mestrado em Ciências Ambientais, Universidade do Estado do Pará (UEPA), Brasil.

${ }^{5}$ Mestre em Ciências Ambientais, Universidade do Estado do Pará (UEPA), Brasil.

Recebido em: 03/10/2016 - Aprovado em: 21/11/2016 - Publicado em: 05/12/2016 DOI: 10.18677/EnciBio_2016B_055

\section{RESUMO}

Os dados de sensoriamento remoto no Infravermelho Termal fornecem medições de fluxos de energia da superfície, como as de Temperatura da Superfície Terrestre (TST). O objetivo deste trabalho foi analisar respostas térmicas de sensor infravermelho termal orbital em diferentes padrões de uso e cobertura no município Mojuí dos Campos, estado do Pará. Para estimar e avaliar os dados de Temperatura de Superfície Terrestre (TST) utilizou-se os softwares PCI Geomática 2015, QGis 2.8 e imagens do sensor Thermal Infrared Sensor (TIRS), a bordo do satélite Landsat-8. Para as análises de TST foram extraídos os valores de TST de 6000 pontos aleatórios, bem como 62077, correspondentes aos centróides do plano de informação de uso e cobertura do solo da área de estudo. Os resultados apontaram maior variabilidade espacial de TST em função da heterogeneidade do uso da terra, principalmente nas áreas de agricultura anual e pasto, com $6,2^{\circ} \mathrm{C}$ e $7,3^{\circ} \mathrm{C}$ acima das suas médias de $33,2^{\circ} \mathrm{C}$ e $31,1^{\circ} \mathrm{C}$, respectivamente. A floresta apresentou comportamento distinto, com temperaturas variando de $28,3^{\circ} \mathrm{C}$ (mínima) a $39,9^{\circ} \mathrm{C}$ (máxima). Comparando os tipos de uso e cobertura do solo e as faixas de temperaturas mais amenas, observa-se que há uma redução da TST em razão da presença de áreas florestais contínuas, em especial a sudoeste da área em análise. Conclui-se que na área em análise a manutenção da cobertura florestal reduz as amplitudes térmicas. Por outro lado, em extensas áreas com pastagem e agricultura anual há maiores variações espaciais da TST.

PALAVRAS-CHAVE: Geotecnologias. Landsat-8. Sensoriamento Remoto. Temperatura de Superfície. 


\title{
ORBITAL IMAGE IN IDENTIFYING WITH THERMAL RESPONSES USE DIFFERENT STANDARDS AND COVERAGE IN THE CITY OF MOJUÍ DOS CAMPOS, STATE OF PARÁ
}

\begin{abstract}
The infrared thermal remote sensing data provides measurements of surface energy fluxes, such as the Land Surface Temperature (LST). The aim of this study was to analyze thermal responses orbital thermal infrared sensor in different patterns of use and coverage in the city Mojuí dos Campos, State of Pará. To assess and evaluate the data of Land Surface Temperature (LST) the softwares used was PCI Geomatics 2015, QGIS 2.8 and images of the Thermal Infrared sensor (TIRS) aboard the Landsat-8 satellite. For the analysis of LST were extracted the LST values of 6000 random points as well as 62077 , corresponding to the centroid of information plan of use and land cover in the study area. The results showed higher spatial variability of LST due to the heterogeneity of land use occurring in the areas of annual crops and pasture, with $6.2^{\circ} \mathrm{C}$ and $7.3^{\circ} \mathrm{C}$ above their average $33.2^{\circ} \mathrm{C}$ and $31.1^{\circ} \mathrm{C}$, respectively. The forest showed different behavior, with temperatures ranging from $28.3^{\circ} \mathrm{C}$ (minimum) to $39.9^{\circ} \mathrm{C}$ (maximum). After compare the ty pes of land use and cover and the tracks of milder temperatures, it is observed that there is a reduction of LST due to the presence of continuous forest areas, especially southwest of the area in question. We conclude that on the area in question the maintenance of forest cover reduces the thermal amplitudes. Moreover, on large areas with annual pasture and agriculture there are larger spatial variations of LST.
\end{abstract}

KEYWORDS: Geotechnology. Landsat-8. Remote sensing. Temperature surface.

\section{INTRODUÇÃO}

Os dados de sensoriamento remoto no Infravermelho Termal (Infrared Thermal - TIR) fornecem medições importantes de fluxos de energia de superfície da terra, que geralmente são chamadas de Temperatura da Superfície Terrestre (TST) (MARTIN \& TOMÁS, 2009). A integração de sistemas de informações geográficas (SIG) e sensoriamento remoto têm sido amplamente aplicados como ferramenta de geotecnologias para detecção do uso do solo e das mudanças de cobertura da terra, bem como para variação de temperatura de superfície terrestre (PAVÃO et al., 2015a).

Neste contexto, a evolução e a utilização da ferramenta sensoriamento remoto, por meio de uma análise da distribuição espacial do uso da terra e temperatura da superfície, constitui-se como uma excelente ferramentas para a elaboração de mapas de áreas de ilhas de calor urbano, como também no auxílio a tomadas de decisões por parte dos órgãos gestores (DOS ANJOS et al., 2013).

$\mathrm{Na}$ Amazônia as pressões (demográficas e econômicas) de diversos agentes - como madeireiros, pecuaristas, grileiros e aqueles que praticam desmatamentos têm impactado diretamente na mudança de temperatura de superfície e, por conseguinte, no clima local (PASSOS, 2011). Neste contexto, a mudança no uso e cobertura do solo tem provocado alterações no balanço de radiação da superfície terrestre (CARDOSO et al., 2009).

DELGADO et al. (2012), afirmam que a substituição das áreas verdes por coberturas como asfalto e concreto influencia nos valores da temperatura, umidade relativa do ar e direção dos fluxos de ventos, principalmente nos anos de anomalias na temperatura e precipitação pluvial, como por exemplo nos anos de forte El Niño. Em áreas urbanas e de solo exposto geralmente são registradas amplas variações 
no valor de temperatura radiante da superfície (ROGAN et al., 2013). Ou seja, mudanças no uso do solo causam um aumento da temperatura da superfície radiante em razão da diminuição da biomassa.

Assim, este estudo teve como objetivo analisar a variação da temperatura de superfície, utilizando sensor infravermelho orbital em diferentes padrões de uso e cobertura no município Mojuí dos Campos, localizado no oeste do estado do Pará. A presente pesquisa envolve a delimitação de zonas de temperatura de superfície em relação à configuração do uso de cobertura do solo, utilizando técnica de sensoriamento remoto.

\section{MATERIAL E MÉTODOS}

O estudo foi realizado no município de Mojuí dos Campos, No Oeste do Estado do Pará. O município pertencente à Mesorregião do Baixo Amazonas, entre os paralelos $03^{\circ} 32^{\prime} 50.27^{\prime \prime}$ e $02^{\circ} 36^{\prime} 59.20^{\prime \prime}$ de lat itude Sul e entre os meridianos de 544ㅇ' 55.50" e 5498'12.44" de longitude Oeste.

Mojuí dos Campos fica próximo aos municípios de Santarém, Prainha, Alenquer, Belterra e Uruará, sendo um dos maiores produtores de grãos do Pará, onde está concentrado cerca de $40 \%$ do arroz, milho e soja produzidos na região oeste. Na Figura 1 fez-se a identificação da área de estudo.

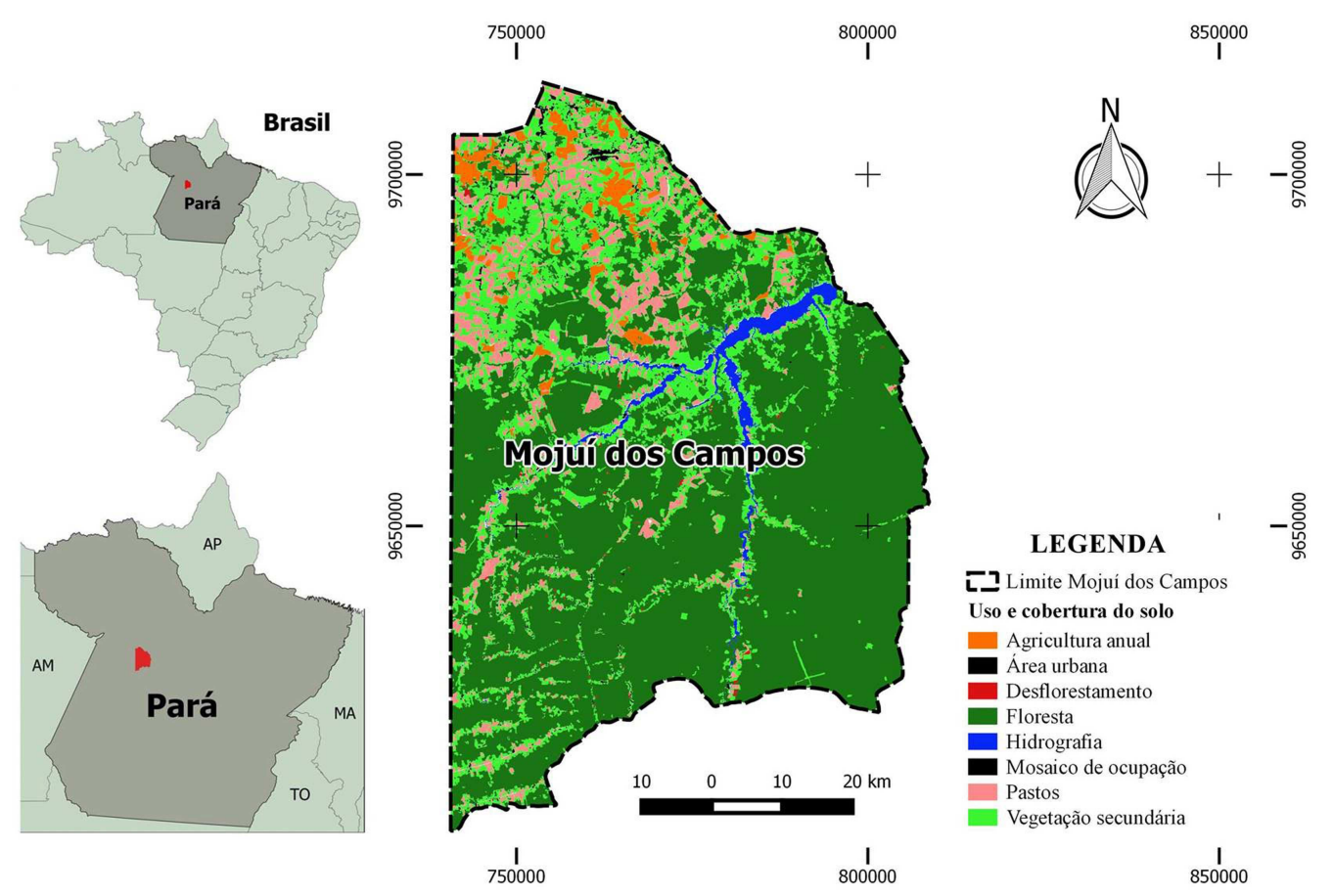

FIGURA 1. Localização do município Mojuí dos Campos, Pará.

Os dados foram obtidos por meio de informações secundárias disponibilizadas nas bases de instituições reconhecidas do Governo Federal, tais como: Instituto Brasileiro de Geografia e Estatística (IBGE); Instituto Brasileiro de Meio Ambiente e Recursos Renováveis (IBAMA), Agência Nacional de Águas (ANA), Instituto Nacional de Pesquisa Espacial (INPE) e Instituto Chico Mendes de Conservação e Biodiversidade (ICMBio). O mapeamento e processamento de todos os dados vetoriais foram realizados no SIG QGis 2.8, versão Wien, iniciando com a adição dos Planos de Informações (PIs) do município de Mojuí do Campos e 
posteriormente o uso e cobertura do solo. Todos os Pls foram projetados para o sistema de coordenadas UTM, Datum SIRGAS 2000, Zona 21 Sul.

Neste estudo foi utilizada a imagem do sensor Thermal Infrared Sensor (TIRS), a bordo do satélite Landsat-8, com duas faixas espectrais no infravermelho termal 10.6 - $11.19 \mu \mathrm{m}$ e 11.5 - $12.51 \mu \mathrm{m}$. A imagem foi obtida gratuitamente no banco de dados do Serviço de Levantamento Geológico Americano (United States Geological Survey - USGS), disponível em http://landsat.usgs.gov/, correspondente a data de passagem de 14/07/2015 às 10h. A imagem selecionada apresentou menor porcentagem de nuvens, correspondendo ao mês de estação seca e ano de forte El Niño. A imagem foi processada no software PCI Geomática 2015 por meio do assistente ATCOR (Focus/Analysis/ATCOR) em seis etapas, conforme os seguintes passos:

- Configuração do sensor de imagem orbital Specifying sensor and image settings;

- Geraram-se "máscaras" de remoção de névoas por meio do comando Haze Removal and Cloud Masking;

- Especificaram-se as condições de altitude e iluminação Specifying elevation and illumination conditions das imagens;

- Fez-se a configuração de visibilidade e reflectância do solo Specifying visibility and ground reflectance;

- Foram realizadas as calibrações radiométricas por meio dos metadados das imagens, e;

- Por fim, estimou-se a temperatura de superfície.

As imagens termais possibilitaram interpretação da distribuição espacial temporal da Temperatura de Superfície Terrestre (TST) (PONZONI, 2012). Os mapas temáticos gerados foram utilizados nas avaliações $e$ análises das informações de TST, sendo a maioria na escala de 1:60.000.

Para as análises de TST foram extraídos no QGis 2.8 os valores de 6.000 pontos aleatórios, bem como 62.077, correspondentes aos centróides do plano de informação $(\mathrm{PI})$ de uso e cobertura do solo disponibilizados pelos projeto TerraClass, referentes ao mapeamento do ano de 2012, onde identificaram-se cerca de 12 classes de uso do solo. Por fim, fez-se uma análise descritiva e interpolação usando Krigagem dos pontos extraídos para avaliar valores de TST mínima, máxima e média e desvios padrões.

\section{RESULTADOS E DISCUSSÃO}

As temperaturas de superfície (TST) no município de Mojuí dos Campos variaram, no momento da passagem do satélite, entre 26,7 a $42,8^{\circ} \mathrm{C}$. Em julho de 2015 a menor e maior temperatura de superfície ocorreu na área de vegetação secundária. Nessa sequência de análise, identificou-se que a temperatura média predominante foi de $30,8{ }^{\circ} \mathrm{C}$. Outra classe de uso e cobertura da terra que se registrou maiores médias de valores de TST foram às áreas destinadas a cultivo anual e pastagem, $\operatorname{com} 6,2^{\circ} \mathrm{C}$ e $7,3^{\circ} \mathrm{C}$ acima de suas médias de $33,2^{\circ} \mathrm{C}$ e $31,1^{\circ} \mathrm{C}$, respectivamente. $\mathrm{Na}$ Tabela 1 , são apresentados os resultados da estatística por região do uso e cobertura da terra em relação aos intervalos de TST, obtendo TST máxima, TST mínima, TST média e desvio padrão predominante na área em análise. 
TABELA 1 - Valores estatísticos de TST do uso e cobertura do uso e cobertura do solo mapeado pelo projeto TerraClass do ano 2012.

\begin{tabular}{|c|c|c|c|c|}
\hline \multirow{3}{*}{ Classes de uso e cobertura do solo } & \multicolumn{4}{|c|}{2015} \\
\hline & \multicolumn{4}{|c|}{ Temperatura de Superfície Terrestre $\left({ }^{\circ} \mathrm{C}\right)$} \\
\hline & Min & Max & Dp & Md \\
\hline Agricultura anual & 30,0 & 39,4 & 1,22 & 33,2 \\
\hline Área urbana & 31,4 & 34,8 & 0,68 & 32,9 \\
\hline Desflorestamento & 29,0 & 37,6 & 1,41 & 31,5 \\
\hline Floresta & 28,3 & 39,9 & 1,43 & 31,5 \\
\hline Hidrografia & 29,5 & 34,7 & 1,07 & 31,7 \\
\hline Mosaico de ocupações & 28,5 & 37,1 & 1,42 & 31,6 \\
\hline Pasto limpo & 28,6 & 38,5 & 1,42 & 32,3 \\
\hline Pasto sujo & 28,8 & 40,0 & 1,31 & 32,7 \\
\hline Regeneração com pasto & 28,7 & 37,9 & 1,32 & 32,6 \\
\hline Vegetação secundária & 26,7 & 40,8 & 1,39 & 31,8 \\
\hline Área não observada & 29,2 & 37,3 & 1,34 & 31,6 \\
\hline Outros & 29,2 & 35,5 & 1,08 & 31,6 \\
\hline Média & 28,9 & 37,8 & 1,33 & 31,8 \\
\hline
\end{tabular}

Nota: Min $=$ Temperatura mínima, Max $=$ Temperatura máxima, $M d=$ Temperatura média e $\mathrm{Dp}=$ Desvio padrão.

A Figura 2 ilustra a imagem termal referente ao mês de junho de 2015, início da estação de baixa oferta pluvial, onde na porção norte, nota-se que há um predomínio de temperaturas entre 32,2 e 34,7 C. A floresta apresentou comportamento distinto, com temperaturas variando de $28,3^{\circ} \mathrm{C}$ (mínima) a $39,9^{\circ} \mathrm{C}$ (máxima). Comparando os tipos de uso e cobertura do solo e as faixas de temperaturas mais amenas, constata-se que há uma redução da TST em razão da presença de áreas florestais contínuas, em especial a sudoeste da área em análise, conforme Figura 2 e 3.

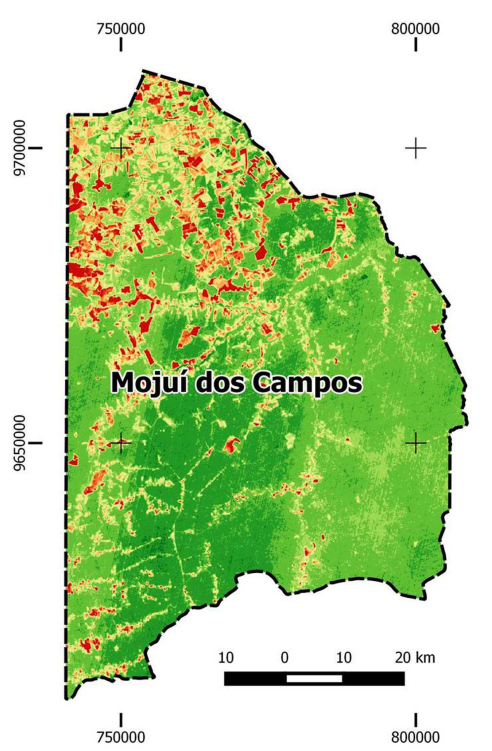

FIGURA 2. Imagem termal referente ao mês de junho de 2015 às $10 \mathrm{~h}$ 36min no Mojuí dos Campos.

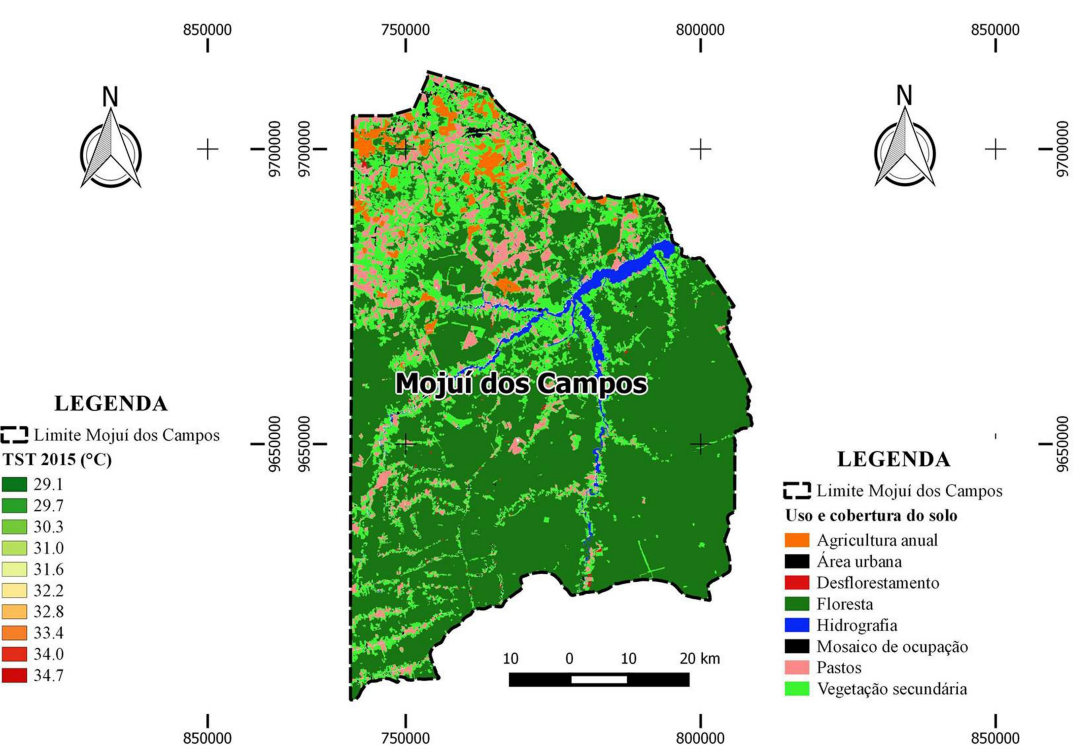

FIGURA 3. Uso e cobertura do solo e TST de 2015 às $10 \mathrm{~h} 36 \mathrm{~min}$ de Mojuí dos Campos.

Na Figura 4 evidencia-se que a área urbana não experimenta uma grande variação TST, devido à natureza seca de materiais urbanos não-evapotranspirativa, 
enquanto que na área com cobertura vegetal (floresta e vegetação secundária) observa-se maior desvio-padrão dos valores de TST $(1,43)$ em comparação com outros tipos de cobertura do solo, indicando a heterogeneidade das copas das árvores (QIN, 2001).

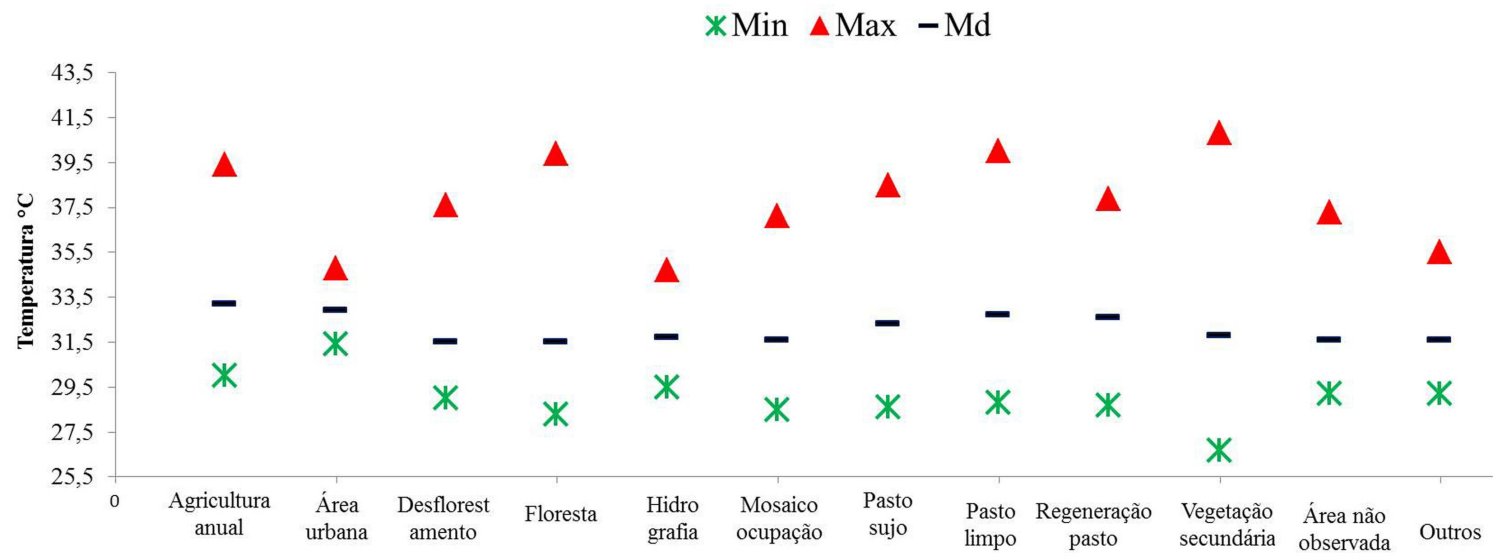

FIGURA 4 - Análise comparativa das médias de TST de 2015 do uso e cobertura do solo.

A Tabela 2 apresenta cinco faixas TST onde se identificou que a agricultura anual com $68,09 \mathrm{~km}^{2}$ ficou sobre uma faixa de TST entre 33,5 a $43,5^{\circ}$ C. A floresta com 1.882,09 km² ficou sobre a faixa de TST entre 30,5 a $32,5^{\circ}$ C. As áreas de pastos ficaram sobre a faixa entre 33,5 a $42,5^{\circ} \mathrm{C}$, correspondendo a uma área total de 232,06 km²; porém, $105,19 \mathrm{~km}^{2}$ de pasto alcançaram TST superiores a $42,5^{\circ} \mathrm{C}$.

TABELA 2 - Faixa de TST do uso e cobertura do uso e cobertura do solo mapeado pelo projeto TerraClass do ano 2012.

\begin{tabular}{|c|c|c|c|c|c|c|}
\hline \multirow{2}{*}{\multicolumn{2}{|c|}{$\begin{array}{l}\text { Classes de uso e } \\
\text { obertura do solo }\end{array}$}} & \multicolumn{5}{|c|}{ Temperatura $\left({ }^{\circ} \mathrm{C}\right)$} \\
\hline & & $29,5-30,5$ & $30,5-32,5$ & $32,5-33,5$ & $33,5-42,5$ & $>42,5$ \\
\hline Agricultura anual & & - & 1,05 & 2,45 & 68,09 & 63,91 \\
\hline Área urbana & & - & & & 4,71 & 1,22 \\
\hline Desflorestamento & & 0,90 & 2,39 & 2,73 & 4,26 & 0,33 \\
\hline Floresta & ลู & 550,82 & $1.822,09$ & 593,53 & 261,73 & 28,95 \\
\hline Hidrografia & & 0,03 & 9,54 & 65,06 & 25,02 & 1,16 \\
\hline Mosaico de ocupações & శూ & 1,92 & 5,88 & 8,92 & 17,01 & 5,21 \\
\hline Pasto limpo & $\frac{\omega}{2}$ & 1,30 & 14,23 & 39,48 & 114,91 & 50,96 \\
\hline Pasto sujo & & 0,17 & 2,57 & 11,90 & & 29,03 \\
\hline Regeneração c & & 0,5 & 4,40 & 12,24 & 51 & 25,20 \\
\hline Vegetação secundária & & 23,44 & 163,97 & 269,77 & 491,23 & 100,22 \\
\hline
\end{tabular}

A Figura 5 ilustra a interpolação e reclassificação dos valores de TST de 6.000 pontos extraídos aleatoriamente da imagem termal. Ao norte observa-se a TST predominante foi de $42,5^{\circ} \mathrm{C}$ (cor vermelha), cor respondendo ao uso e ocupação de agricultura anual, área urbana e pasto. Ao sul concentrou-se a área de menor valor de TST, predominante com 29,5 ${ }^{\circ}$, indic ando os efeitos térmicos expressos nas áreas que possuem significativa cobertura de vegetação primária. $\mathrm{Na}$ Figura 6 observar a variação de TST, aumentando no sentido da área florestal para a área urbana. 


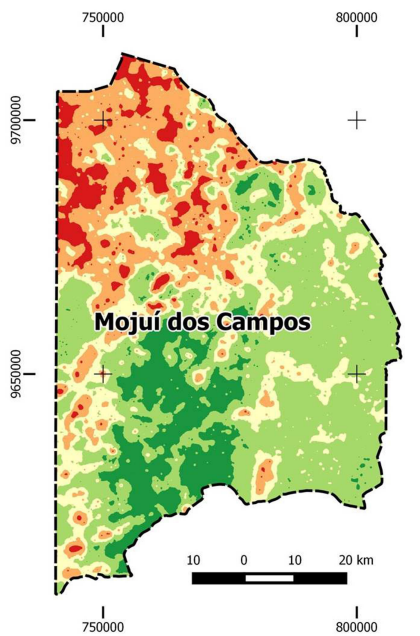

FIGURA 5. Temperatura de superfície de 2015 às $10 \mathrm{~h} 36 \mathrm{~min} d \mathrm{e}$ Mojuí dos Campos.

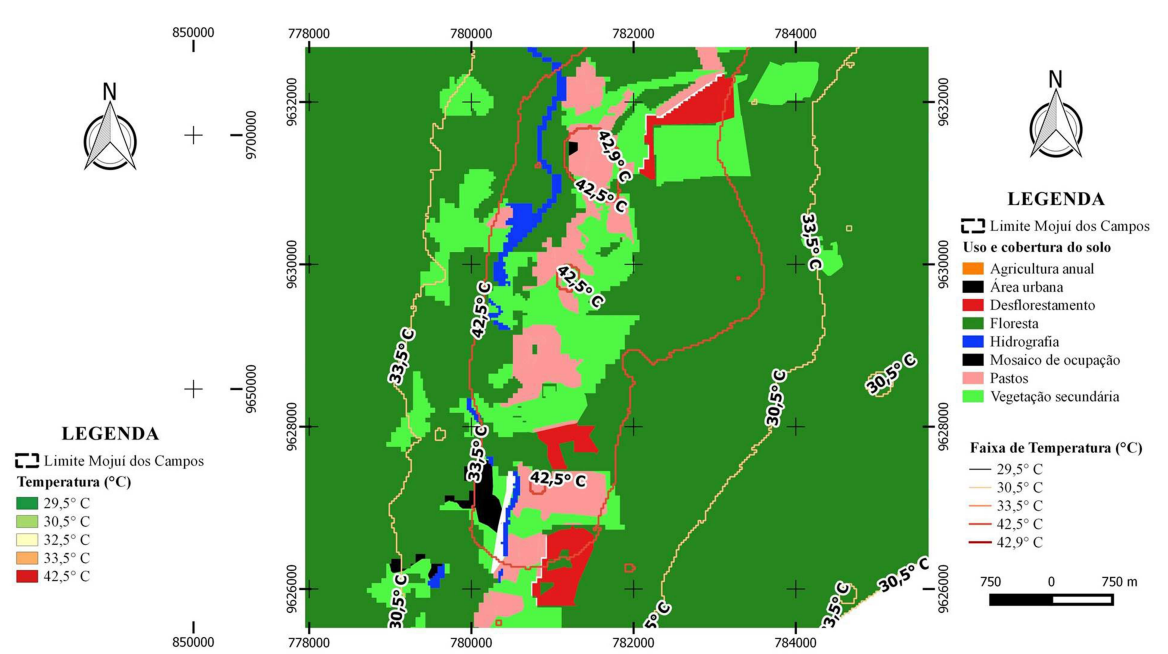

de Figura 6. Faixa de Temperatura de superfície de sobre o Uso e cobertura do solo de Mojuí dos Campos.

Com base nos resultados, aferiram-se variações de TST em razão da mudança do padrão do tipo de uso e cobertura do solo, influenciando diretamente nos valores de temperatura, com menores valores em florestas e maiores em áreas urbanas e agricultura anual, principalmente ao norte da área em análise. De acordo com AUMOND \& COMIN (2012), os elevados valores de TSTs podem influenciar na umidade relativa do ar e na direção dos fluxos de ventos, bem como provocar mudanças nas condições microclimáticas. Com o aumento da TST o conforto ambiental é afetado pelo estresse térmico e possui influência direta dos elementos do clima como o vento, a chuva, a umidade relativa do ar, radiação solar e temperatura (BERTONCELLI et al., 2013).

As altas temperaturas, no mês de julho às $13 \mathrm{~h}$ na classe de floresta, podem estar associadas também ao reduzido estoque de água na vegetação e absorção de parte da radiação solar incidente durante o processo de evapotranspiração e fotossíntese (PRIMAVESI et al., 2007; COSTA et al., 2013). Quanto às áreas agrícolas, com ou sem vegetações esparsas, têm contribuído para uma ampla variação no valor de TST, reforçando a observação de que as temperaturas vinculadas às regiões recobertas por vegetação florestal resultaram em temperaturas mais brandas (FERREIRA, 2011). Ou seja, menores amplitudes térmicas, indicando que nas áreas com floresta a energia é mantida no sistema solofloresta-atmosfera.

PAVÃO et al. (2015b), observaram áreas que apresentam baixa ou nenhuma densidade de vegetação,altos valores de temperatura da superfície, haja vista que a energia disponível ao ambiente é utilizada prioritariamente para aquecimento do solo e do ar adjacente à superfície. As temperaturas extremas nas áreas de agricultura anual e pastos podem provocar redução de manancial, erosão, assoreamento e perda de agricultura, prejudicando a produção de alimentos e 0 abastecimento local e regional (SANTOS et al., 2012). Portando as perdas na cobertura vegetal, principalmente na porção nordeste pode comprometer o microclima, como por exemplo, as extensas áreas com pastagens e cultivos anuais quanto à manutenção da cobertura florestal reduzem as amplitudes térmicas (SANTOS et al., 2016).

Assim, o conhecimento de limites térmicos sobre o uso e cobertura do solo de uma determinada região, com rigor de detalhes, são de extrema importância nos ENCICLOPÉDIA BIOSFERA, Centro Científico Conhecer - Goiânia, v.13 n.24; p.593 2016 
estudos climáticos, pois eles podem apresentar diferenças de microclimas oriundas da morfologia da superfície (COELHO \& CORREA, 2013). Nesse sentido, as variações de TST na área em estudo evidenciam modificações nos padrões espaciais e temporais dos elementos do clima da região, principalmente em área sem cobertura vegetal que é responsável pela regulação da quantidade de energia no processo de aquecimento da superfície terrestre (PILLAR, 1995; LEITE, 2011; FIALHO, 2012).

\section{CONCLUSÃO}

As informações de temperatura da superfície do canal infravermelho termal do sensor contribuíram na identificação, espacialização e compreensão da condição térmica da área em estudo. Os resultados mostraram que o desenvolvimento da área urbana e solo exposto têm elevado a TST, ou seja, as mudanças no uso do solo causam um aumento da temperatura da superfície em razão da redução da biomassa.

Observou-se que as alterações do uso e ocupação do solo - principalmente a substituição de florestas para solo exposto, cortes e novas plantações - foram significativas para as condições térmicas no município de Mojuí dos Campos.

Por fim, infere-se que a temperatura superficial, extraída a partir das informações das ferramentas do sensoriamento remoto, pode subsidiar a avaliação de aspectos de TST de extensas regiões, servindo, portanto, como estudo base, de baixo custo e análise rápida para pesquisas científicas e diretrizes de políticas públicas que possam mitigar alterações térmicas e mudanças climáticas a nível local.

\section{REFERÊNCIAS}

AUMOND, J. J.; COMIN, J. J. Abordagem sistêmica e o uso de modelos para recuperação de áreas degradadas. Revista Árvore, v.36, n.6, p. 1099-1118, 2012.

BERTONCELLI, P.; MARTIN, T. N.; ZIECH, M. F.; PARIS, W.; CELLA, P. S. Conforto Térmico Alterando a Produção Leiteira. Enciclopédia Biosfera, Centro Científico Conhecer - Goiânia, v.9, n.17, 2013.

CARDOSO, M. R. D.; MARCUZZO, F. F. N.; PINTO FILHO, R. F. Caracterização Física da Bacia do Rio Araguaia visando determinar susceptibilidade a enchentes. Anais. XIV Simpósio Brasileiro de Geografia Aplicada. Universidade Federal de Viçosa, 2012.

COELHO, A. L.; CORREA, W. S. Temperatura de Superfície Celsius do Sensor TIRS/Landsat-8: Metodologia e Aplicações. Revista Geografia Acadêmica. v.7, n.1, p.1678-7226, 2013.

COSTA, P. F.; SAPIENZA, J. A. Avaliação da necessidade de correção radiométrica para comparação de Índice de Vegetação por Diferença Normalizada (NDVI). Anais XVI Simpósio Brasileiro de Sensoriamento Remoto - SBSR. Foz do Iguaçu. INPE, 2013. 
DELGADO, R. C.; RODRIGUES, R. A.; FARIA, A. L. L.; PESSÔA, C. S.; DAHER, M. Uso do sensoriamento remoto na estimativa dos efeitos de ilhas de calor. Revista Brasileira de Ciências Ambientais, n. 25, p. 70 - 80, 2012.

DOS ANJOS, A. W.; DELGADO, R. C.; OLIVEIRA JÚNIOR, J. F.; DE GOIS, G.; MORAES, N. O. Temperatura da Superfície Continental Associada aos Eventos Meteorológicos na Cidade do Rio de Janeiro, Rj. Enciclopédia Biosfera, Centro Científico Conhecer - Goiânia, v.9, n.17, 2013.

FERREIRA, H. A. A Percepção e o contexto no desenho de estratégias de adaptação à mudança climática no uso agrícola das terras. Dissertação (mestrado) - Universidade Federal de Santa Catarina, Centro de Ciências Agrárias, Programa de Pós-Graduação em Agroecossistemas, Florianópolis, 2011. Disponível em:< http://repositorio.ufsc.br/xmlui/handle/123456789/95379>. Acesso em 14 set. 2016.

FIALHO, E. S. Estudos Climáticos em Sítios Urbanos e Rurais,(Orgs.) SILVA, Charlei Aparecido da e FIALHO Edson Soares, Concepções e Ensaios da Climatologia Geográfica e-book. ED. UFGD. PP-83-112, 2012.

INPE e EMBRAPA. TerraClass Levantamento de informações de uso e cobertura da terra na Amazônia. Disponível em acesso em 15 de agosto de 2016.

LEITE, R. C. V.; FROTA, A. B. A Influência da Mudança nos Padrões de Ocupação do Solo Sobre a Ventilação Natural Urbana: Estudo de caso na cidade de Fortaleza, Ceará. Anais. XIV Encontro Nacional de Tecnologia do Ambiente Construído ENTAC. Juiz de Fora. 2012.

MARTIN, H.; TOMÁS, K. The influence of topography on the forest surface temperature retrieved from Landsat TM, ETM + and ASTER thermal channels. ISPRS Journal of Photogrammetry and Remote Sensing, n. 64, p. 585-591. 2009.

PAVÃO, V. M.; QUERINO, C. A.; BENEDITTI, C. A.; PAVÃO, L. L.; QUERINO, J. K.; MACHADO, N. G.; BIUDES, M. S. Temperatura e Albedo da Superfície por Imagens TM Landsat 5 em Diferentes Usos do Solo no Sudoeste da Amazônia Brasileira. Revista Brasileira de Climatologia, v. 16, p. 169 -183, 2015.

$\mathrm{PCl}$. Geomatica OrthoEngine: User Guide Version 15. Ontario, Canada: PCI Geomatics Enterprises Inc., Jan 2015. 168p.

PILLAR, V. D. Clima e vegetação. UFRGS, Departamento de Botânica. 1995. Disponível em http://ecoqua.ecologia.ufrgs.br. Acesso em: 10 ago. 2016.

PONZONI, F. J. Sensoriamento Remoto da Vegetação. 2. Ed. Atualizada e ampliada - São Paulo: Oficina de Texto, 2012.

PRIMAVESI O.; ARZABE C.; PEDREIRA M. S. Mudanças climáticas: visão tropical integrada tropical, São Carlos, SP, Embrapa Pecuária Sudeste, 2007. 
QIN, Z.; KARNIELI, A.; BERLINER, P. A mono-window algorithm for retrieving land surface temperature from Landsat TM data and its application to the Israel-Egypt border region. International Journal of Remote Sensing, Londres, v. 22, n. 18, p. 3719-3746, 2001.

ROGAN, J.; ZIEMER, M.; MARTIN, D.; RATICK, S.; CUBA, N.; DELAUER, V. The impact of tree cover loss on land surface temperature: A case study of central Massachusetts using Landsat Thematic Mapper thermal data. Applied Geography, Amsterdam, v. 45, p. 49-57, 2013.

SANTOS, J. S.; SILVA, V. P.; SILVA, E. R.; ARAÚJO, L. E.; COSTA, A. D. Campo Térmico Urbano e a sua Relação com o Uso e Cobertura do Solo em Cidade Tropical Úmida. Revista Brasileira de Geografia Física, v. 5, n. 3, p. 540-557, 2012.

SANTOS, L. S.; MARTORANO, L. G.; BATALHA, S. S. A.; PONTES, A. N.; JÚNIOR, O. M. S.; DOS SANTOS WATRIN, O.; GUTIERREZ, C. B. B. Imagens Orbitais e Termografia Infravermelho na Avaliação da Temperatura de Superfície em Diferentes Usos e Cobertura do Solo na Floresta Nacional do Tapajós e seu Entorno, PA. Revista Brasileira de Geografia Física, 9(4), 2016.

USGS - Geological Survey / Serviço de Levantamento Geológico Americano (2013). Aquisição de imagens orbitais digitais gratuitas do satélite Landsat-8: data de passagem 14/07/2015. Acesso em 15 mar. 2016. Disponível em: $<$ http://landsat.usgs.gov>. 